\title{
Menschliche Sprachfähigkeit und ihre neurobiologischen Korrelate. Reflexionen aus glottodidaktischer Sicht
}

\author{
A glottodidactic perspective on human language faculty and its neurobiological correlates
}

\author{
Barbara SADOWNIK \\ Uniwersytet Marii Curie-Skłodowskiej w Lublinie/ Marie Curie-Sklodowska \\ University in Lublin \\ E-mail: barbara.sadownik@wp.pl,
}

\begin{abstract}
The focus in the present contribution is on glottodidactics researchers' attempts at working out a coherent model of foreign language acquisition in its both cognitive and neurobiological aspects. It is argued that the latest developments in cognitive linguistics as well as in psycholinguistics do offer an elaborate account of the nature and the structure of human language capacity, and, as such, they can constitute a source of inspiration for glottodidactics research. By contrast, despite significant achievements over the last decades, our knowledge of the language-mind interface is still elementary, speculative, and does not prove to be significantly relevant for glottodidactics.
\end{abstract}

Keywords: Glottodidactics, Foreign Language Acquisition Theory, Human Language Faculty, Universal Grammar, Cognitive Neurosciences, Neuroimaging

\section{Einleitung}

Das Hauptziel der glottodidaktischen Forschung, das schon Mitte der achtziger Jahre des 20. Jahrhunderts formuliert wurde, ist die Ausarbeitung eines kohärenten theoretischen Modells des Fremdsprachenerwerbs, das vom konkreten Sprachlerner ausgeht. Der vorliegende Beitrag soll zeigen, dass die wichtigsten Impulse für die Erstellung einer Theorie des Fremdsprachenerwerbs in erster Linie von der kognitiven Linguistik und Psycholinguistik, weniger von den kognitiven Neurowissenschaften (Cognitive Neurosciences) gehen, da das Wissen über die tatsächlichen Relationen zwischen Gehirn und Sprache trotz aller bemerkenswerten Erkenntnisse immer noch elementar ist. Die Glottodidaktik wird von wachsenden Erkenntnissen der kognitiven Neurowissenschaften erst in Zukunft profitieren, da sie heutzutage trotz massiver finanzieller Unterstützung, keine innovativen Lösungen anbieten, eher widersprüchliche Konzeptualisierungen und spekulative Interpretationen.

\section{Problemaufriss}

Die Glottodidaktik betrachtet nach wie vor als ihr spezielles Erkenntnisziel, die vielfältigen Dimensionen unterrichtlichen Lernens und Lehrens von Fremdsprachen sys- 
tematisch zu beschreiben und zu erklären, und zwar im Hinblick auf den Sprachlernenden. Als zentrale Komponente der Glottodidaktik ist der Sprachlerner anzusehen, der als Subjekt des Lern- und Objekt des Lehrprozesses ist. Mit anderen Worten, die polnische Glottodidaktik ist anthropologisch orientiert und legt in ihrem Forschungsprogramm ein besonderes Augenmerk auf den Menschen in der Funktion des Sprachlerners und Sprachlehrers (vgl. z.B. F. Grucza 2006). Die Erforschung der konstitutiven Eigenschaften (Sprach(erwerbs)fähigkeiten, Sprachverarbeitungsfähigkeiten, Sprachwissen, etc.), die den Handlungen der Sprachlerner zugrunde liegen und demzufolge als bestimmte Faktoren der generativen Ausstattung (Tiefenstruktur) dieser Objekte/Subjekte aufzufassen sind, wird als eine zentrale Aufgabe der Glottodidaktik definiert. Der Begründer dieser Forschungsdisziplin Franciszek Grucza betont in seinen zahlreichen Arbeiten, dass die Tiefenstruktur der Forschungsobjekte von der Glottodidaktik nur „mittels einer Theorie” erreicht werden kann (F. Grucza/ H.J. Krumm/ B. Grucza 1993: 60). Ein besonderes Kennzeichen der Glottodidiaktik ist die interdisziplinäre Ausrichtung der Forschungsarbeit.

Die Einschätzung des Erkenntnisinteresses am (Fremd)Spracherwerb hängt in erster Linie vom Vorverständnis über Natur, Struktur und Funktionsweise menschlicher Sprache ab (F. Grucza 1997, 2002, 2010). Die Sprache ist zum einen als eine mentale Eigenschaft, d.h. konstitutive Eigenschaft des menschlichen Geistes gekennzeichnet, die auf einer bestimmten Beschreibungs- und Explikationsebene zum Gegenstand der philosophischen, linguistischen, bzw. psycholinguistischen Reflexionen geworden ist, zum anderen als Resultat neurobiologischer Prozesse des Gehirns. Zu allen Zeiten haben Forscher versucht, die mentalen, abstrakt-strukturellen und neuralen Grundlagen der Sprache und im Zusammenhang damit die Organisationsprinzipien des Geistes und Gehirns zu erfassen.

Die Sprachfähigkeit bzw. die linguo-kognitve Fähigkeit (facultas linguarum) als ein Spezifikum des homo sapiens ist ohne Zweifel an seine neurobiologischen Voraussetzungen gebunden. Die Annahmen über die Sprachfähigkeit, wonach sie eine autonome Komponente der mentalen Struktur darstellt und die Annahmen über ihre neurobiologischen Korrelate sind zwei komplementäre, nicht aufeinander reduzierbare Annahmen, die in ihrer wechselseitigen Ergänzung zum Verständnis eines (Fremd)Sprachenerwerbsphänomens im Ganzen jedoch notwendig sind.

Die Linguisten und Psycholinguisten haben inzwischen die menschliche Sprache und ihre einzelnen Komponenten sowie Spracherwerbs- und Sprachverarbeitungsprozesse sehr präzise mit Hilfe von Theorien und Modellen beschrieben und erklärt. Das ist ein unschätzbarer Vorteil. Die von der Linguistik geleistete Beschreibung und Erklärung der Mechanismen der menschlichen Sprachbeherrschung und der sie strukturierenden Prinzipien ist von grundsätzlicher Bedeutung für die Glottodidaktik. Gerade in der Linguistik werden immer mehr testbare Hypothesen aufgestellt, die es ermöglichen sehr viel theoriebasierter an zentrale Fragestellungen heranzugehen als in anderen Domänen der Forschung. In der Generativen Linguistik unter dem Einfluss Noam Chomskys, die eine prominente aktuelle Forschungsrichtung darstellt, sind inzwischen gute und zunehmend verbesserte Theorien abstrakt-struktureller Aspekte 
der menschlichen Sprache erstellt worden, die nicht nur Linguisten weltweit beeinflusst haben. N. Chomsky (2016: 48) schreibt dazu:

Der Perspektivenwechsel hin zur generativen Grammatik im Rahmen des biolinguistischen Ansatzes Mitte des 20. Jahrhunderts öffnete den Weg für eine viel weiter reichende Erforschung nicht nur der Sprache selbst, sondern auch verwandter Gebiete.

Die kognitiven Neurowissenschaften dagegen, die als Hilfsmittel zum Verständnis der Sprach(erwerbs)fähigkeit angesehen werden, stehen eigentlich erst am Anfang des Erkenntnisgewinns. Sie haben inzwischen zahlreiche Versuche unternommen, u.a. linguistische und psychologische Phänomene mit der Neuroanatomie und den neurophysiologischen Funktionen in Verbindung zu bringen. (vgl. dazu ausführlich B. Sadownik 2010). Das ist ein ungeheuer kompliziertes Unterfangen. Die unvorstellbare strukturelle Komplexität des Gehirns macht es schwierig, seine Bestandteile mit bestimmten sprachlichen Funktionen in Zusammenhang zu bringen.

Das entscheidende Problem besteht darin, dass die Neurowissenschaftler noch weit davon entfernt sind, eine nur grobe Skizze einer Gehirntheorie zu entwerfen (N. Chomsky 2000a, 2002) ${ }^{1}$. Die bisherigen Fortschritte der kognitiven Neurowissenschaften lassen sich im Verständnis sprachlicher Leistungen des Menschen weniger mit großen Entwicklungssprüngen ihrer Theoriebildung als vielmehr mit bedeutenden technischen Entwicklungen, insbesondere auf dem Gebiet des Neuroimaging, in Verbindung setzen. (B. Sadownik 2012). Was Neurobiologen bis heute also deutlich fehlt, sind die entscheidenden theoretischen Bausteine des Gehirns. Mit anderen Worten, zu den grundsätzlichen Problemen der Neurowissenschaften gehört, dass es keine kohärente Theorie des Gehirns gibt, an der sich spracherwerbliche Studien in der Glottodidaktik orientieren könnten. Es gibt auch keine klare Vorstellung davon, wie eine solche Theorie des Gehirns erstellt werden könnte.

\section{Die Sprachfähigkeit als Gattungsmerkmal des homo sapiens}

Die Untersuchung der menschlichen Natur sowie der Natur und Struktur von Sprache war bis zur Neuzeit ein Arbeitsfeld der Sprachphilosophie (vgl. P. Hacker 2017). Erst seit dem 19. Jahrhundert gehört die Frage nach dem Aufbau und Funktion der menschlichen Sprache vorwiegend zum Forschungsgegenstand der Linguistik. Linguisten von heute befassen sich mit der Sprache aus zwei gegensätzlichen Forschungsansätzen.

(1) Die gebrauchsbasierten Linguisten sehen Sprache als direktes Resultat von Eigenschaften der gesamten menschlichen Kognition und Bedürfnissen in der Kommunikation. Der Terminus usage based theories wurde in dieser Tradition zuerst von Ronald W. Langacker (1987/1991) verwendet. Inzwischen ist er u.a. von Michael Tomasello $(2003,2004,2005,2009,2011,2015)$ vielfältig angereichert und ausgearbeitet worden. M. Tomasello (2011:333) vertritt konsequent den Standpunkt, dass „Sprachen innerhalb der Beschränkungen bereits existierender menschlicher Kognition und

\footnotetext{
${ }^{1}$ Vgl. dazu auch u.a. A.D. Friederici/ R. C. Berwick/ N. Chomsky/ J. J. Bolhuis (2013), Evolution, brain and the nature of language. (in:) „Trends in Cognitive Sciences” 17, 89-98.
} 
Sozialität" geschaffen wurden. Laut M. Tomasello gibt es keine spezifische Sprach(erwerbs)fähigkeit, die sich von anderen komplexen kognitiven Leistungen prinzipiell unterscheidet. Auch N. Chomsky'sche Hypothese einer angeborenen Universalgrammatik lässt sich für ihn derzeit nicht kohärent formulieren (M Tomasello 2004: 642-644). Die Annahme einer Universalgrammatik hält M. Tomasello für unnötig, weil sich der Spracherwerb durch allgemeinere Lernprozesse und die Entwicklung sozial-kognitiver Fähigkeiten (wie zum Beispiel das Etablieren gemeinsamer Aufmerksamkeit, um auf ein Objekt zu referieren, oder die Fähigkeiten, Intentionen der anderen Akteure zu erfassen) erklären lasse. P. C. Trettenbrein fast in seinem Artikel „The "grammar" in Universal Grammar: A biolinguistic clarification“ M. Tomasellos kritische Auffasung von der Universalgrammatik wie folgt zusammen:

Tomasello has repeatedly attacked the concept of UG because he thinks the existence of a genetically endowed FL to be unnecessary, implausible, or even impossible (2005). Along these lines, he has also argued that UG in fact constitutes an unfalsifiable hypothesis (Tomasello, 2004) and has recently pronounced UG "dead" (Tomasello, 2009). He himself favours accounts of language acquisition and learning that he labels as "usage-based approaches" (Tomasello 2005, p. 191) which, according to him, involve only "one set of general cognitive processes" (Tomasello, 2005, p. 193) that are not domain-specific so that language development is guided only by semantic and pragmatic cues during acquisition. As Tomasello is a quite prominent scientist, his ideas on UG deserve consideration and simultaneously urge refutation (P.C. Trettenbrein 2015: 2).

M. Tomasello stellt sich die Sprache als völlig unspezifisch vor, und zwar, als viele andere Kulturprodukte, im Sinne der Fertigkeiten und Motivationen individueller und geteilter Intentionalität. Von besonderer Bedeutung ist eine bestimmte Art gemeinsamer Aufmerksamkeit oder gemeinsamen Hintergrunds zwischen dem Kommunizierenden und dem Empfänger, die die Grundlage geteilten Verstehens darstellt, auf der sprachliche Konventionen ausgewählt und verstanden werden. In M. Tomasellos Worten:

Die Sprache, oder besser die sprachliche Kommunikation, ist daher nicht irgendeine Art von formalem oder sonstigem Gegenstand, vielmehr ist sie eine Form gesellschaftlichen Handelns, konstruiert durch gesellschaftliche Konventionen, um gesellschaftliche Zwecke zu erreichen, welche zumindest auf einem gewissen geteilten Verstehen und geteilten Zielen der Benutzer beruhen (M. Tomasello 2011: 363).

Das Schlüsselwort für diesen linguistischen Ansatz ist Variation, die nicht nur deskriptiv festgehalten wird. Sie verlangt auch danach, im Hinblick auf ihre Abhängigkeit von verschiedenen grammatikinternen und/oder -externen Einflussfaktoren erklärt zu werden. Die Grammatikforschung erfährt, so M. Konopka (2018: 152), ,eine empirische Wende, in der Variation grammatischer Strukturen zu einem der zentralen Themen wird." Bei einer nur generell festgelegten grammatischen Struktur kann es immer wieder zu im Einzelnen abweichenden Realisierungen kommen. Es geht darum, so (S. Conrad 2010: 227-228), zunächst überhaupt die Variation zu diagnostizieren und dann zwischen in bestimmten Kontexten typischen und untypischen Varianten zu unterscheiden (vgl. S. Conrad 2010). Die Dichotomien richtig/falsch, akzeptabel/nicht akzeptabel etc. sind laut M. Tomasello (2014) nicht mehr zentral, sondern 
es geht um unter bestimmten Umständen mehr oder weniger Wahrscheinliches. Die usage-based theories of language acquisition thematisieren demnach den Erwerb sprachlicher Handlungsfähigkeit von der jeweiligen kommunikativen und sprachlichen Erfahrungsbasis her (vgl. M. Tomasello 2003).

(2) Demgegenüber tendiert die Generative Grammatiktheorie in ihrer von Noam Chomsky (z.B.: 1981, 1982, 1986, 1988, 1995, 2005, 2011, 2016) geprägten Version dazu „Variation nicht als konstituierendes, sondern als marginales Phänomen menschlicher Sprache anzusehen“ (J. Meisel/ Ch. Schwarz 2002: 437). N. Chomsky antwortete in einem Interview mit B. Aarts (2001: 5) auf die Frage "What is your view of modern corpus linguistics?“ mit der Feststellung: „It doesn’t exist. If you have nothing, or if you are stuck, or if you are worried about Gothic, then you have no choice." Für N. Chomsky selbst als Vertreter des galileischen Stils, der immer nur theoretisch über das Sprachwissen (knowledge of language) gearbeitet hat, sind Universalgrammatik und Sprachgebrauch strikt getrennt. Genetisch verankerte Sprachfähigkeit lässt sich laut N. Chomsky durch konkrete Sprachdaten weder stützen noch widerlegen, da sie zum tatsächlichen Sprachgebrauch gehören (T.W. Fitch/ M.D. Hauser /N. Chomsky 2005). Sprache gilt laut N. Chomsky nicht als empirisches Faktum: eine solche Empirie bzw. empirische Resultate, die ein hohes Maß an Variationen im Spracherwerb aufweisen, sind ohnehin unbedeutend. Die menschliche Sprach(erwerbs)fähigkeit im Sinne N. Chomskys lässt sich an sprachlichen Performanzdaten mit all ihren Zufälligkeiten, Sprechereigentümlichkeiten, situativen Besonderheiten nicht ablesen. Sie ist - so die Kritiker von N. Chomsky, wie z. N. Evans/S. C. Levinson (2009: 429-430), S. Müller (2016: 455) - ,gegenüber empirischer Falsifizierung weitgehend immunisiert.“ N. Chomskys Ansatz stellt die Frage der Angeborenheit von Sprach(erwebs)fähigkeit sowie deren mögliche neuronale Implementierung eindeutig in den Vordergrund.

Es unterliegt keinem Zweifel, dass Noam Chomsky ein besonders einflussreiches Modell für die Sprache, für ihre Grammatik, sowie für den von ihm in den Mittelpunkt gestellten Sprecher und Hörer der Sprache entwickelt hat. Die Generative Grammatik hat zu einer fundamentalen Revision der Vorstellungen über die Natur der menschlichen Sprachfähigkeit geführt. Die generative Prinzipien- und Parametertheorie, deren ursprüngliche Konzeption, die Rektions- und Bindungstheorie (N. Chomsky 1981, 1982, 1986) war, ist zwar in den vergangenen Jahren mehrmals stark modifiziert und als Minimalistisches Programm (N. Chomsky 1995) weiterentwickelt worden, aber die dabei aufgeworfenen Fragestellungen beschäftigen die Linguistik bis in die Gegenwart hinein. Sprache ist aus der Sicht der Generativisten ein Wissenssystem besonderer Natur, das im menschlichen Geist verankert ist. Der mentalistischen Sicht zufolge ist die Untersuchung der Sprachfähigkeit im tiefsten Sinne ein Studium des menschlichen Gehirns auf einer abstrakten Ebene. Das ist gemeint, wenn N. Chomsky (z.B. 1982, 1986, 1988) die Linguistik als Teil der Psychologie bzw. der Humanbiologie oder Biolinguistik versteht.

Die mentalistische Sicht ist ein eher naturwissenschaftlicher Ansatz zur Untersuchung der Sprachfähigkeit. Dabei geht es darum, die menschliche Sprachfähigkeit naturwissenschaftlich korrekt zu charakterisieren, und zwar, in ihren bei der Geburt im 
Menschen angelegten Strukturen und in den entfalteten Strukturen im erwachsenen Menschen, der eine bestimmte Sprache oder mehrere Sprachen spricht. Die zentrale Frage der Generativen Linguistik ist es, welche Form das der Sprachfähigkeit des Menschen zugrundeliegende Wissenssystem hat, wobei das Wissen um die Sprachstruktur als Kern der Sprachfähigkeit angesehen wird. Wenn man die Regeln, die die Grammatik einer Sprache ausmachen, nicht nur adäquat beschreiben will, sondern darüber hinaus erklären will, warum sie so sind, wie sie sind, stellt laut N. Chomsky die biologische Organisation des Geistes unserer Art eine adäquate Begründungsinstanz dar. N. Chomsky (1982: 107) schreibt dazu: „Grammars have to have a real existence, that is, there is something in your brain that corresponds to the grammar. That has got to be true".

Der Begriff "Grammatik" wird hier nicht im traditionellen Sinn verstanden, sondern bezeichnet eine Theorie über (abstrakte) mentale Repräsentation. N. Chomsky vertritt damit eine biologische Deutung des Grammatikbegriffs. Die angeborene formale Kompetenz zum Erwerb und zur Repräsentation von Grammatiken ist für ihn ein aufgabenspezifisches System, ein mentales Organ, analog zur visuellen Perzeption. Die eigentliche Novität des generativen Forschungsprogramms liegt nun keineswegs darin, dass für den grammatischen Bereich ein zugrundeliegendes System mentaler Repräsentation angenommen wird. Vielmehr steht hinter dem generativen Programm die Auffassung, dass die interne Grammatik (I(nternalized)-Grammatik, d.h. das qua mentaler Repräsentation im Gehirn real existierende Objekt, das eigentliche Forschungsobjekt der Grammatiktheorie ist (N. Chomsky 1986: 21-22). Die I-Sprache ist dagegen nichts anderes als die empirische Datenbasis, die dem Linguisten den Zugriff auf die I-Grammatik erlaubt, da ein direkter Zugang offensichtlich nicht möglich ist: „The I-Language (...) is some element of the mind of the person who knows the language, acquired by the learner and used by the speaker-hearer" (N. Chomsky 1986: 220). N. Chomsky betont, dass Aussagen zur I-Sprache keineswegs direkt für die die Ebene des neuronalen Substrats zu formulieren sind, sondern auf einer höheren Ebene, welche von den spezifischen Mechanismen des Gehirns, also von den Details der neuronalen Schaltungen, abstrahiert. Die I-Sprache ist laut N. Chomsky ein Derivat der I-Grammatik und nicht etwa umgekehrt. Demgegenüber ist aus traditioneller Perspektive die externalisierte Sprache (E-Sprache) das eigentliche Forschungsobjekt für viele Linguisten. Die E-Grammatik ist nichts anders als die Menge deskriptiver Aussagen über die E-Sprache, d.h. eine abstrakte Funktion, die die Elemente der E-Sprache aufzählt. Als E-Sprache bezeichnet N. Chomsky die Totalität der Äußerungen, die in einer Sprachgemeinschaft gemacht werden können, also die Produkte der Sprache im Sinne der aktuell oder potentiell gebildeten Sätze, inklusive sprachlicher Handlungen und Verhaltensweisen. Interessant ist die Tatsache, dass der Terminus der »E-Sprache«, der auch von N. Chomsky stammt, für ihn beinahe inhaltsleer war, „da ich E-Sprache als alles, was nicht I-Sprache ist, definierte“ (N. Chomsky 2016: 40).

N. Chomsky spricht in seinen Arbeiten häufig von der ,language faculty“ und meint damit jeden Bereich der menschlichen Kognition, in dem die spezifischen grammatischen Prinzipien und Gesetzmäßigkeiten verankert sind. „Language faculty“ 
ist in diesem Sinne mit der I-Grammatik gleichzusetzen. Besonderes Interesse kommt dem Teil der I-Grammatik zu, der als angeboren angesehen wird. Er ist allen Menschen gemeinsam und somit allen Grammatiken möglicher (natürlicher) menschlicher Sprachen. Deshalb nennt man diesen Teil der I-Grammatik auch Universalgrammatik, wobei I-Sprache, wie oben angedeutet, ein Derivat der I-Grammatik ist. Chomsky vertritt konsequent seine Auffassung dass es sich bei der I-Sprache um eine individuelle Sprache handelt, da sie im Individuum als eine Komponente des menschlichen Geistes repräsentiert ist. In seinem Buch, dessen Originalausgabe unter dem Titel What Kind of Creatures Are We? 2016 bei Columbia University Press erschien, argumentiert N. Chomsky (2016: 41) wie folgt:

Ich werde meine Aufmerksamkeit hier auf die I-Sprache beschränken, eine biologische Eigenschaft des Menschen, eine Subkomponente (vor allem) des Gehirns, ein Organ des Geistes/Gehirns in dem unpräzisen Sinn, in dem das Wort »Organ« in der Biologie verwendet wird. Ich verstehe den »Geist« hier als das Gehirn, auf einer gewissen Stufe der Abstraktion betrachtet. Dieser Ansatz wird manchmal als das biolinguistische Programm bezeichnet. Er wird weithin als kontrovers betrachtet, obwohl ich keinen Grund dafür erkennen kann.

An dieser Stelle soll erwähnt werden, dass im Minimalistischen Programm von N. Chomsky (1995) die denknotwendigen minimalistischen Voraussetzungen gesucht und postuliert werden, aus denen dann deduziert wird, wie die sprachliche Welt beschaffen sei, weil diese ja nicht anders sein könne als deren perfekte Implementierung. Über die postulierte Erklärungsadäquatheit seiner Grammatiktheorie hinaus soll aufgezeigt werden, warum die Sprache die Eigenschaften hat, die sie hat; es geht um Natürlichkeit, Schönheit und Eleganz, Einfachheit und Ökonomie, so dass die Anforderungen eines kognitiven Systems, mit dem die Sprachfähigkeit interagiert, getroffen werden. Sprache ist für N. Chomsky (1995: 385-386) ein ,perfect system, meeting external constraints as well as can be done" und eine solche Auffassung wird als „strong minimalist thesis“ bezeichnet. N. Chomsky nimmt in dem Minimalistischen Programm an, dass die Sprachfähigkeit im Sinn einer angeborenen, genetisch kodierten Universalgrammatik minimal sein muss. Ihre einzige Aufgabe besteht darin, sprachliche Ausdrücke anhand eines sehr einfachen Berechnungssystems zu generieren, die phonetisch und semantisch in jeweils eigenen externen Systemen interpretiert werden (N. Chomsky 2006).

Mit anderen Worten, zur Sprachfähigkeit im engeren Sinne gehört ausschließlich das formale Berechnungssystem der Syntax; funktionale Aspekte einschließlich der Semantik-Pragmatik, aber auch die Morpho-Phonologie gehören nicht dazu. Das Berechnungssystem, das rein syntaktischer Natur ist, garantiert eine kontextfreie rekursive Verkettungsoperation und eine Kopieroperation. Erstere Operation generiert komplexe sprachliche Ausdrücke aus einfacheren, wobei nur Elemente mit zueinander passenden Merkmalen verkettet werden, letztere sorgt dafür, dass sprachliche Elemente an verschiedenen Positionen im Satz auftreten können. Eine weitere Operation garantiert, dass nur Elemente mit zueinander passenden Merkmalen verkettet werden (N. Chomsky 2000). Das syntaktische Berechnungssystem interagiert im Minimalistischen Programm zwar mit anderen kognitiven Systemen, ist allerdings diesen aber 
vorgelagert und arbeitetet als autonomes Modul (vgl. dazu auch R.C. Berwick et al. 2013). Anders gesagt, die syntaktische Komponente bleibt im Minimalistischen Programm weiterhin zentral. Notwendig sind laut N. Chomsky insbesondere Schnittstellen, damit andere kognitive Komponenten auf die sprachlichen zugreifen können. Die Schnittstelle zum artikulatorisch-perzeptiven System sorgt beispielsweise dafür, dass Sprache gehört und gesprochen werden kann. Der Ausdruck von Gedanken läuft über eine Schnittstelle mit dem konzeptuell-intentionalen System. Sowohl das artikulatorisch-perzeptuelle Lautsystem als auch das konzeptuell-intentionale Bedeutungssystem werden als externe Systeme aufgefasst (vgl. dazu N. Chomsky 2004). Es ist wichtig zu betonen, dass im Minimalistischen Programm die Spracherwerbsfähigkeit noch stärker in die Gehirnarchitektur eingebunden und mental-biologisch interpretiert wird (R. Berwick/ N. Chomsky 2011).

\section{Auf der Suche nach den neurobiologischen Korrelaten der Sprachfähigkeit}

Die neurale Basis der Sprache ist immer noch ein weitgehend ungelöstes Problem. Wissenschaftler stehen eigentlich erst am Anfang des Verständnisses dieser komplexen Wechselwirkungen zwischen Sprache und Gehirn. Aus der Sicht der Humanbiologie bzw. Biolinguistik wird kaum bestritten, dass die Sprachfähigkeit des Menschen auf spezifischen hirnphysiologischen Strukturen beruht, die etwa bei anderen Organismen in dieser Funktion nicht vorhanden sind. Die genauen neuroanatomischen Grundlagen des sprachlichen Wissenssystems liegen jedoch noch im Dunkeln. In anderen kognitiven Teilsystemen, so z.B. im visuellen Bereich, ist die Forschung in Bezug auf die physikalischen Grundlagen viel weiter als bei der Untersuchung der Sprachfähigkeit.

Schon vor mehr als 150 Jahren wurden zwei Sprachareale in der linken Hirnhälfte entdeckt, das Broca-Areal im Frontallappen für das Sprechen und das Wernicke-Areal im Temporallappen für das Verstehen von Sprache und sie gelten bis heute als konstitutive Sprachzentren. Im letzten Jahrhundert sind viele Neurolinguisten zu der Ansicht gekommen, dass die Sprachfähigkeiten nicht auf diese beiden klassischen Zentren beschränkt. Inzwischen hat man entdeckt, dass auch vordere Bereiche des Temporallapenns sowie etliche Bereiche des Frontallappens (interior, anterior sowie posterior) und der untere Parietallappen bei der Sprachverarbeitung eine Rolle spielen (A.D. Friederici/ S.M.E. Gierhan 2013). Die wichtigsten zugrundeliegenden hirnfunktionellen und hirnstrukturellen Voraussetzungen der rezeptiven und produktiven Sprachverarbeitung im voll entwickelten Sprachverarbeitungssystem sind mittlerweile relativ gut erforscht und beschrieben, obwohl es keine plausible Erklärung gibt, wie die Relation zwischen Gehirnstrukturen und Sprachrezeption bzw. -produktion genau beschaffen ist (vgl. z.B. W. L. Schuerman et. al. 2017).

Die neurobiologischen Korrelate der Etablierung der Sprachfähigkeit im sich entwickelnden Gehirn während des Spracherwerbsprozesses sind bislang nur in Ansätzen verstanden. Aktuelle Forschungsarbeit hat auch einen sensomotorischen Schaltkreis für Sprache im hinteren linken Schläfenlappen identifiziert, von dem man vermutet, dass er zwischen Spracherkennung und Sprachproduktion vermittelt. Dieser Schaltkreis ist an der Sprachentwicklung beteiligt (A.D. Friederici 2012). Heutzutage sind 
jedoch noch viele scheinbar einfache spracherwerbliche Fragen aus neurobiologischer Sicht weiterhin ungelöst.

Umstritten ist immer noch die Frage, inwieweit eine direkte Korrelation zwischen konkreten, von der Generativen Linguistik postulierten Prinzipien und Gesetzmäßigkeiten und physiologischen Gegebenheiten des Gehirns besteht (R.C. Berwick/ N. Chomsky 2011, R.C. Berwick et al. 2013; s. auch J. J. Bolhuis et al.). Auch Vorstellungen darüber, wie ein physikalisches bzw. biologisches System mentale Prozesse hervorbringt, sind nur rudimentär (vgl. M. Bennett et al. 2010). Es wird lediglich spekuliert, dass mentale sprachliche Phänomene auf der physikalisch groben Ebene von Neuronen und Synapsen eine Erklärung finden könnten. Aus der Auffassung, dass mentale Leistungen auf Eigenschaften des Gehirns beruhen, folgt nicht, dass sie auf der Basis von Konzepten und Befunden der gegenwärtigen Neurophysiologie erklärbar sind. Zwar lassen sich heute Entsprechungen von mentalen Phänomenen zu neurobiologischen Prozessen finden - wie etwa das Feuern von Neuronen in bestimmten Hirnbereichen. Es wäre aber ein Missverständnis, die derzeit verfügbare Befunde als Erklärungen für mentale Phänomene zu betrachten. Im Gegenteil, sie vergrößern den Erklärungsbedarf, weil zusätzlich noch erklärt werden muss, welche physikalischen Prinzipien in welcher Weise mentale Prozesse hervorrufen, warum die mentalen Phänomene gerade mit diesen oder jenen neurobiologischen Prozessen korrelieren. Vieles von dem, was über Veränderungen in Struktur und Funktion des Gehirns während der sprachlichen Entwicklung geschrieben wird, muss als einleuchtende Spekulation verstanden werden. Die Erwartungen sind enorm, die Forschungserkenntnisse werden dagegen als relativ bescheiden ausgewertet. Dieser Unzulänglichkeit sind sich insbesondere die ganz renommierten Wissenschaftler bewusst, die sich wie beispielsweise der deutsche Molekularbiologe, der Nobelpreisträger Bert Sackmann (2009: 1) dazu wie folgt äußern:

Es gibt einen riesigen Haufen von Einzelbefunden, das ist überhaupt keine Frage. Das Gebiet floriert, kein Zweifel, aber ich glaube nicht, dass es da einen Durchbruch gibt. (...) aber ich halte das auch ein bisschen für Windmacherei (B. Sackmann 2009: 1)2.

Mit der Entwicklung der Bildgebung bzw. der bildgebenden Verfahren eröffneten sich in den letzten Jahren neuartige, nicht-invasive vielversprechende Möglichkeiten zur Erforschung der Gehirnstrukturen und Sprachfunktionen am lebenden Menschen (vgl. dazu B. Hüsing, et al. 2006, A.M B. de Groot, P. Hagoort, 2018). Die enge Verflechtung des Gehirns mit der Sprache macht jedoch die Suche nach den neurobiologischen Korrelaten langwierig und kompliziert. „Hirnforscher faszinieren uns mit ihren Fragen“ schreibt F. Hasler (2013: 5) in seinem Buch „Neuromythologie“, "aber sie vertrösten uns regelmäßig mit den Antworten“. In der einschlägigen Fachliteratur wird übereinstimmend hervorgehoben, dass jede der modernen bildgebenden Techniken ihre ganz spezifischen Stärken und Schwächen hat, die sich gegenseitig ergänzen

\footnotetext{
2 So konstatierte Bert Sackmann in seinem öffentlichen Vortrag „Die Neurophysiologie von Entscheidungen" an der Jacobs Universität in Bremen am 23.02.2009. Er erhielt 1991 gemeinsam mit dem Biophysiker Erwin Neher den Nobelpreis für Physiologie und Medizin.
} 
und nicht ersetzen. Wenn man doch die Fortschritte der Hirnforschung genauer betrachtet, so findet man ihren Grund in der Entwicklung der Technik, allem voran der digitalen Technologien, und nicht im erweiterten Wissen über die zugrunde liegenden Prozesse im Gehirn. Zum gegenwärtigen Zeitpunkt werden Methoden des Neuroimaging ausschließlich im Rahmen von kontrollierten Studien und Forschungsverfahren eingesetzt die ausschließlich eine Beschreibung aber noch keine wissenschaftliche Erklärung im Bereich der neurobiologischen Organisation der Sprache liefern können. Mit anderen Worten, es gibt aber eine enorme Erklärungslücke zwischen der Beschreibung der Hirnaktivität auf der neurophysiologischen Ebene und der theoretischen Modellierung der kognitiven Leistungen des Gehirns. Auch wenn anhand bildgebender Verfahren kognitive und emotionale Leistungen des Gehirns „lokalisiert“ werden können, gelingt es den Hirnforscher offensichtlich nicht, die „Semantik“ der biologischen Kodierung zu verstehen (L. Jäncke 2005). Die Lücke zwischen den vorhandenen Daten und dem Verständnis ihrer Bedeutung, die wir durch Rückschlüsse überwinden müssen, ist unvorstellbar gewaltig groß (vgl. ausführlich dazu P. Hagoort 2017). Aus diesem Grund ist ein gewisses Maß an kritischer Distanz auch gegenüber den ,anschaulichen“ Ergebnisse mit bildgebenden Verfahren angebracht (vgl. dazu B. Sadownik 2012).

In seiner Besprechung der Messdaten über das Gehirn, die mit immer neuen bildgebenden Methoden angehäuft werden, zog N. Chomsky (2000) auch eher eine enttäuschende Bilanz. Dabei bezieht er sich vor allem auf die Schlüsse aus den Ergebnissen ereigniskorrelierter Potentiale (ERPs). Für ihn gibt es in der Generativen Linguistik ein ziemlich gutes Verständnis der Unterschiede zwischen Arten der Abweichungen von normalen Strukturen sprachlicher Gebilde, die jetzt als Abweichungen von dem einen oder anderen der in der Sprachbegabung verankerten Prinzipien abstrakter Struktur verstanden werden könnten. Über die ereigniskorrelierten Messungen hinaus hätten neuere empirische Beobachtungen von elektrischen Aktivitäten des Gehirns Korrelate zu einigen dieser linguistisch charakterisierten Abweichungstypen gefunden. Sie hätten sogar gezeigt, dass semantische und syntaktische Regel-Verletzungen unterschiedliche elektrophysiologische Reaktionen aufweisen. All diese Beobachtungsresultate lieferten laut N. Chomsky noch keine Erklärung. Da N. Chomsky nur an systematisch fundierten explanatorischen Erkenntnissen interessiert ist, sind ihm die bisherigen Ergebnisse bloße Merkwürdigkeiten, und zwar deswegen, weil ihr Erklärungswert aufgrund des Fehlens einer einheitlichen Theorie der elektrischen Aktivität des strukturierten Gehirns unklar ist. Da wir eine solche Theorie nicht haben, argumentiert N. Chomsky in seinen Arbeiten, kennen wir die Gründe nicht, warum genau diese Resultate auftreten und nicht andere.

Bis jetzt wurde konsequent die Ansicht vertreten, dass der Nachweis neuroanatomischer Entsprechungen bzw. Korrelate zur Universalgrammatik noch aussteht.

\section{Ein wirklicher Durchbruch?}

Nun versucht die Germanistin und Neuropsychologin Angela D. Friederici in einem umfassenden Werk ,Language in Our Brain. The Origins of a Uniquely Human Capacity“. Vorwort von Noam Chomsky, 2018 erschienen im renommierten Verlag des 
Massachusetts Institute of Technology (MIT) in Cambridge zu erklären, wie die an der Sprache beteiligten Hirnrindenregionen zusammenspielen - und wie innerhalb von Millisekunden aus Lauten Wörter, Wortgruppen, Sätze und letztlich Inhalte entstehen. In ihrem Buch über die neurobiologischen Grudlagen der Sprache verweist A.D. Friederici auf eine tief im Gehirn liegende Faserverbindung, deren Entdeckung durch sie persönlich und ihr Forschungsteam hohe Wellen in der Welt der Sprachforschung schlug: Dabei handle es sich um ein Faserbündel, den sogenannten Fasciculus Arcuatus im Gehirn zwischen dem Broca-Areal und dem Wernicke-Areal. A.D. Friedrich betont, dass die Faserbündel $\mathrm{m}$ Gegensatz zu den schon lange bekannten Spracharealen tief im Gehirn und nicht in der Hirnrinde sitzen ${ }^{3}$. Diese Verbindung ist laut A.D. Friederici eine Art Datenautobahn, auf der Informationen zwischen den sprachrelevanten Hirnarealen transportiert werden, und damit die entscheidende Struktur für die Verarbeitung der Grammatik, der eigentlichen Grundlage von Sprache. Mit anderen Worten, laut A.D. Friederici wurden 2018 erste empirische Beweise vorgelegt, die auf das Vorhandensein eines Organs hindeuten, das für die Universalgrammatik zuständig ist. Über eigene Entdeckung spricht A. D. Friederici in einem Interview mit S. Kara (2018) wie folgt:

Es ist schon lange bekannt, dass Grammatik und Wörter an getrennten Orten im Gehirn verarbeitet werden. Für die Grammatik ist das sogenannte Broca-Areal zuständig, für die Wörter das Wernicke-Areal. Wir haben uns die Verbindungen zwischen diesen beiden Regionen angesehen. Unsere Hypothese war, dass ein bestimmtes Nervenfaserbündel, welches das Broca-Areal mit dem Wernicke-Areal verbindet, verantwortlich sein müsste für die Verarbeitung der Syntax, also der Regeln für die Kombination von Wörtern.

Der sogenannte Fasciculus Arcuatus ist im Gehirn aller Erwachsenen weltweit deutlich ausgeprägt und variiert nur minimal, je nachdem in welcher Sprache eine Person aufgewachsen ist. Damit ist, so argumentiert A.D. Friederici (2018), ein tief liegendes Faserbündel der neurowissenschaftliche Beleg für die Idee von N. Chomsky, nach der allen Menschen ein universelles System von Prinzipien für Grammatik, d.h. die Universalgrammatik angeboren ist. Diese Hirnstruktur ist bei Erwachsenen sehr ausgereift, dagegen ist sie bei anderen Primaten und Kleinkindern laut A.D. Friederici wenig vorhanden, entsprechend gering sind es auch ihre sprachlichen Fähigkeiten. Jede neue Idee löst immer auch Emotionen aus. Es sieht so aus, dass A.D. Friederici mit ihrem Engagement und ihren leidenschaftlichen Erklärungen auch N. Chomsky selbst für diese Idee gewonnen hat: „Er fand es sehr spannend, dass es für seine Theorie erste Evidenzen gibt. Er hatte ja diese theoretische Idee schon in den sechziger Jahren, er ist aber kein Empiriker" (A.D. Friederici 2018: 2) . In seinem Vorwort zu „Language in Our Brain“ gibt sich N. Chomsky (2018) von dieser Idee

\footnotetext{
${ }^{3}$ Vgl. dazu das erste differenzierte neurologische Modell der Sprache, das sog. WernickeGeschwind-Modell, das auf den Fasertrakt Fasciculus arcuatus veweist, d.h. auf eine „Einbahnstraßen-Verbindung“, und zwar vom Wernicke- zum Broca-Areal. Ist die Verbindung (Fasciculus arcuatus) zwischen diesen beiden Teilen des Gehirns gestört, so bezeichnet man dies als Leitungsaphasie. Bei dieser sind sowohl das Sprachverständnis als auch die Sprachproduktion intakt (N. Geschwind 1970, 1972).
} 
überzeugt und argumentiert, dass diese Hirnstruktur sich offenbar entwickelt hat, um der menschlichen Fähigkeit zu dienen, Grammatik zu verarbeiten, dem Kern der menschlichen Sprachfähigkeit.

Zeichnet sich in der Forschung ein echter Durchbruch bei der Suche nach den neurobiologischen Korrelaten der Sprachfähigkeit ab?

\section{Bibliografie}

Aarts, B. (2001), Corpus linguistics, Chomsky and fuzzy tree fragments. In: Ch. Mair/ M. Hundt (Hrsg.), Corpus linguistics and linguistic theory. Amsterdam, 5-13.

Bennett, M./ P. Hacker/ J.R. Searle/ D. Dennett (2010), Neurowissenschaft und Philosophie: Gehirn, Geist und Sprache. Frankfurt/ M.

Berwick, R.C./ N. Chomsky (2011), The Biolinguistic Program: The Current State of its Development. In: A.M. Di Sciullo/ C. Boeckx (Hrsg.), Biolinguistic Investigations. Oxford, 19-41.

Berwick, R.C./ P. Pietroski/ B. Yankama/ N. Chomsky (2011), Poverty of the Stimulus Revisited. In: „Cognitive Science”, 35, 1207-1242.

Berwick, R.C./ A.D. Friederici/ N. Chomsky/ J.J. Bolhuis (2013), Evolution, brain, and the nature of language. In: „Trends in Cognitive Science” 17, 89-98.

Berwick, R.C./ N. Chomsky (2016), Why Only Us. Cambridge.

Bolhuis, J.J./ I. Tattersall/ N. Chomsky/ R.C. Berwick (2014), How could language have evolved?. In: „PLOS Biology” 12 (8): e1001934.

Bolhuis, J.J./ I. Tattersall/ N. Chomsky/ R.C. Berwick (2015), Language: UG or Not to Be, That Is the Question. In: „PLOS Biology”13 (2): e1002063.

Chomsky, N. (1981), Lectures on Government and Binding. The Pisa Lectures. Dordrecht.

Chomsky, N. (1982), The generative enterprise A discussion with Riny Huybregts and Henk van Riemsdijk. Dordrecht.

Chomsky, N. (1984), Modular approaches to the study of the mind. San Diego.

Chomsky, N. (1986), Knowledge of Language: its Nature, Origin and Use. New York.

Chomsky, N. (1988), Language and Problems of Knowledge. The Managua Lectures. Cambridge, Mass.

Chomsky, N. (1995), The Minimalist Program. MA/London, Cambridge Mass.

Chomsky, N. (1996). Powers and Prospects. Reflections on Human Nature and the Social Order. London.

Chomsky, N. (2000a), New Horizons in the Study of Language and Mind. Cambridge. Chomsky, N. (2000b), The Architecture of Language. Oxford.

Chomsky, N. (2000c), Minimalist inquiries: The framework. In: R. Martin/ D. Michaels/ J. Uriagereka (Hrsg.) Step by step: Essays on minimalist syntax in honor of Howard Lasnik. Cambridge, 89-155.

Chomsky, N. (2002), On Nature and Language. Cambridge.

Chomsky, N. (2005), Three factors in language design. In: „Linguistic Inquiry”, 36 (1), 1-22. 
Chomsky, N. (2006), Approaching UG from below, (in: U. Sauerland/ H.-M. Gärtner, (Hrsg.), Interfaces + recursion = language? Chomsky's minimalism and the view from syntax-semantics. Berlin/ New York. 1-29.

Chomsky, N. (2011), Language and other cognitive systems: What is special about language?, (in): „Language Learning and Development”, 7(4), 263-278.

Chomsky, N. (2013), Problems of projection. „Lingua” 130, 33-49.

Chomsky, N. (2016), Was für Lebewesen sind wir?, Berlin.

Conrad S. (2010), What can a corpus tell us about grammar?. In: A. O'Keeffe/ M. McCarthy (Hrsg.), The Routledge handbook of corpus linguistics (Routledge Handbooks in Applied Linguistics). London/New York. 227-240.

De Groot, A.M.B./ P. Hagoort (2018), Research methods in psycholinguistics and the neurobiology of language: A practical guide. Oxford.

Evans, N./ S.C. Levinson (2009), The myth of language universals: Language diversity and its importance for cognitive science, „Behavioural and Brain Sciences” 32, 429-492.

Fitch, T.W./ M.D. Hauser/ N. Chomsky (2005), The evolution of the language faculty: Clarifications and implications. In: „Cognition” 97, 179-210.

Friederici A.D. (2011), The brain basis of language processing: From structure to function. In: „Physiological Reviews” 91, 1357-1392.

Friederici A.D. (2012), The cortical language circuit: From auditory perception to sentence comprehension. In: „Trends in Cognitive Sciences” 16, 262-268.

Friederici A. (2018), Language in Our Brain. The Origins of a Uniquely Human Capacity. Vorwort von Noam Chomsky. Cambridge.

Friederici A.D./ R.C. Berwick/ N. Chomsky/ J.J. Bolhuis (2013), Evolution, brain and the nature of lang,uage. In: „Trends in Cognitive Sciences”17, 89-98.

Friederici A.D./ S.M. Gierhan,(2013), The language network. In: „Current Opinion in Neurobiology" 23 (2), 250-254.

Geschwind, N. (1970), The organization of language and the brain. In: „Science” $170,940-944$.

Geschwind, N. (1972), Language and the brain, „Scientific Americian” 226, 76-83.

Grewendorf, G. (1994), Interview with Noam Chomsky: Notes on linguistics and politics. In: „Linguistische Berichte” 153, 386 -392.

Grucza, F. (2002), Zu den Forschungsgegenständen der Linguistik und der Glottodidaktik. Zum Wesen menschlicher Sprachen und zu ihren Funktionen. In: H. Barkowski/ R. Faistauer (Hrsg.), in Sachen Deutsch als Fremdsprache. Baltmannsweiler, 231-244.

Grucza, F. (2006), Glottodydaktyka: nauka - praca naukowa - wiedza. In: „Glottodydaktyka", 20,19-40.

Grucza, F. (2010), Główne tezy antropocentrycznej teorii języków. In: „, Lingwistyka Stosowana/Applied Linguistics/Angewandte Linguistik" 2. 41-68.

Grucza, F./ H.J. Krumm/ B. Grucza (1993), Beiträge zur wissenschaftlichen Fundierung der Ausbildung von Fremdsprachenlehrern. Warszawa.

Hacker, P. (2017), The Passions: A study of Human Nature. Oxford. 
Hagoort, P. (2017), The core and beyond in the language-ready brain . In: "Neuroscience \& Biobehavioral Reviews" 81, 194-204.

Haxler, F. (2013), Neuromythologie. Eine Streitschrift gegen die Deutungsmacht der Hirnforschung,3. Ausg., Bielefeld.

Hüsing B./ L. Jäncke/ B. Tag (2006), Impact Assessment of Neuroimaging. Zürich.

Jäncke, L. (2005), Methoden der Bildgebung in der Psychologie und den kognitiven Neurowissenschaften. Stuttgart.

Kara, S. Universalgrammatik: "Ich denke, dass er denkt, dass ...". In: ZEIT ONLINE. https://www.zeit.de/2018/18 [abgerufen am 2. Mai 2018]

Konopka, M. (2018), Korpuslingustik, Grammatiktheorie, GrammatikographieI. In: A. Wöllstein/ P. Gallmann/ M. Habermann /M. Krifka (Hrsg.) Grammatiktheorie und Empirie in der germanistischen Linguistik. Berlin, 151-185.

Langacker, R. (1987), Foundations of Cognitive Grammar. Theoretical prerequisites. Bd. 1 Stanford.

Langacker, R. (1991), Foundations of Cognitive Grammar. Bd 2. Stanford.

Meisel J./ Ch. Schwarz (2002), Romanistische und theoretische Linguistik. Das Besondere und das Allgemeine. In: „Romanische Forschungen” 114, 423-444.

Müller, H. M. (2013), Psycholinguistik - Neurolinguistik. München.

Müller S. (2016), Grammatical theory: From transformational grammar to constraintbased approaches. Berlin.

Sadownik, B. (1997), Glottodidiaktische und psycholinguistische Aspekte des Fremdsprachenerwerbs Lernerperspektive. Lublin.

Sadownik, B. (2010), Modulare Architektur der menschlichen Sprachfähigkeit-kognitive und neurobiologische Dimensionen, Lublin.

Sadownik, B. (2012), Möglichkeiten und Grenzen des Einsatzes bildgebender Verfahren bei der Erforschung neurobiologischer Grundlagen der Sprache. Eine Spezifizierung aus glottodidaktischer Perspektive. In: M. Olpińska-Szkiełko/ S. Grucza/ Z. Berdychowska/ J. Żmudzki (Hrsg.), Der Mensch und seine Sprachen. Frankfurt a.M., 500-514.

Schuerman, W.L., et al., (2017), Mapping the speech code: Cortical responses linking the perception and production of vowels, ,Frontiers in Human Neuroscience” 11, 161-183.

Tomasello, M. (2003), Constructing a Language: A Usage-Based Theory of Language Acquisition. Cambridge.

Tomasello, M. (2004), What kind of evidence could refute the UG hypothesis?. In: „Studies in Language” 28, 642-644.

Tomasello, M. (2006), Die kulturelle Entwicklung des menschlichen Denkens. Zur Evolution der Kognition. Frankfurt a. M.

Tomasello, M. (2009), Universal Grammar is dead. In: „Behavioral and Brain Sciences" 32(5), 470-471.

Tomasello, M. (2011), Ursprünge der menschlichen Kommunikation. Frankfurt.

Tomasello, M. (2014), Eine Naturgeschichte des menschlichen Denkens. Berlin.

Trettenbrein, P.C., (2015), The "grammar" in Universal Grammar: A biolinguistic clarification „Questions and Answers in Linguistics” 2 (1), 1-10. 